\title{
Will AI write scientific papers in the future?
}

\section{Yolanda Gil}

University of Southern California

\section{Correspondence}

Yolanda Gil, University of Southern California, California, USA.

Email: gil@isi.edu

Delivered on February 9, 2020 at the Thirty-Fourth AAAI Conference on Artificial Intelligence (AAAI-20) in New York, NY, USA

\section{Funding information}

the National Science Foundation; the Defense Advanced Research Projects Agency; the National Institutes of Health; the Intelligence Advanced Research Projects Activity; the National Aeronautics and Space Administration; the Air Force Office of Scientific Research; the Office of Naval Research; Elsevier; the Kavli Foundation

\begin{abstract}
In this presidential address, I would like to start with a personal reflection on the field and then share with you the research directions I am pursuing and my excitement about the future of AI. In my personal research to advance AI while advancing scientific discoveries, one question that I have been pondering for some years now is whether AI will write scientific papers in the future. I want to reflect on this question, and look back at the many accomplishments in our field that can make us very hopeful that the answer will be yes, and that it may happen sooner than we might expect.
\end{abstract}

\section{A PERSONAL PERSPECTIVE ON AI}

My first AAAI conference was in 1986. Just 2 weeks prior, I had landed in Pittsburgh from my home country, Spain. I found AAAI to be a very inspiring community. In my view, AI researchers have been visionary, broad, inclusive, interdisciplinary, determined, and successful at challenging endeavors. It is a very vibrant field, and I think the AAAI conference is where I see that breadth, interdisciplinarity, and determination at its best. And I am very proud to be part of this AI community that has been tackling profound challenges, and I am often amazed by what it has accomplished as I look back over the years.

In the 80s, when I arrived at Carnegie Mellon University, there was a course on architectures for intelligence, where I learned so much about the breadth of ideas in approaching intelligence. Allen Newell was working with others on the SOAR architecture, adopting cognitive models of intelligence. Jaime Carbonell, who was my advisor, was doing research on engineering diverse intelligent capabilities for learning, reasoning, and meta-reasoning, all within the same Prodigy framework. Tom Mitchell was working on THEO, which was a sophisticated frame system to organize general knowledge and facts about the world. Geoff Hinton, who was at CMU at the time, was working on backpropagation as an alternative basis for intelligent architectures. In that course, we also learned about different architectures for intelligence that were being explored elsewhere. Roger Schank at Yale emphasized how intelligence is demonstrated in telling interesting stories that prompt others to respond with a good follow-up question or a related and equally interesting story. Rod Brooks at MIT was investigating subsumption architectures, with basic capabilities controlling sensors and actuators, and then on those capabilities you build more elaborate ones that demonstrate higher intelligence. All this provided a very broad view on the approaches to study intelligence and on the field of AI. I hope that students today continue to seek these kinds of opportunities to appreciate the breadth of our field.

Over the decades, I have seen tremendous accomplishments from our community in all areas of AI. Figure 1 


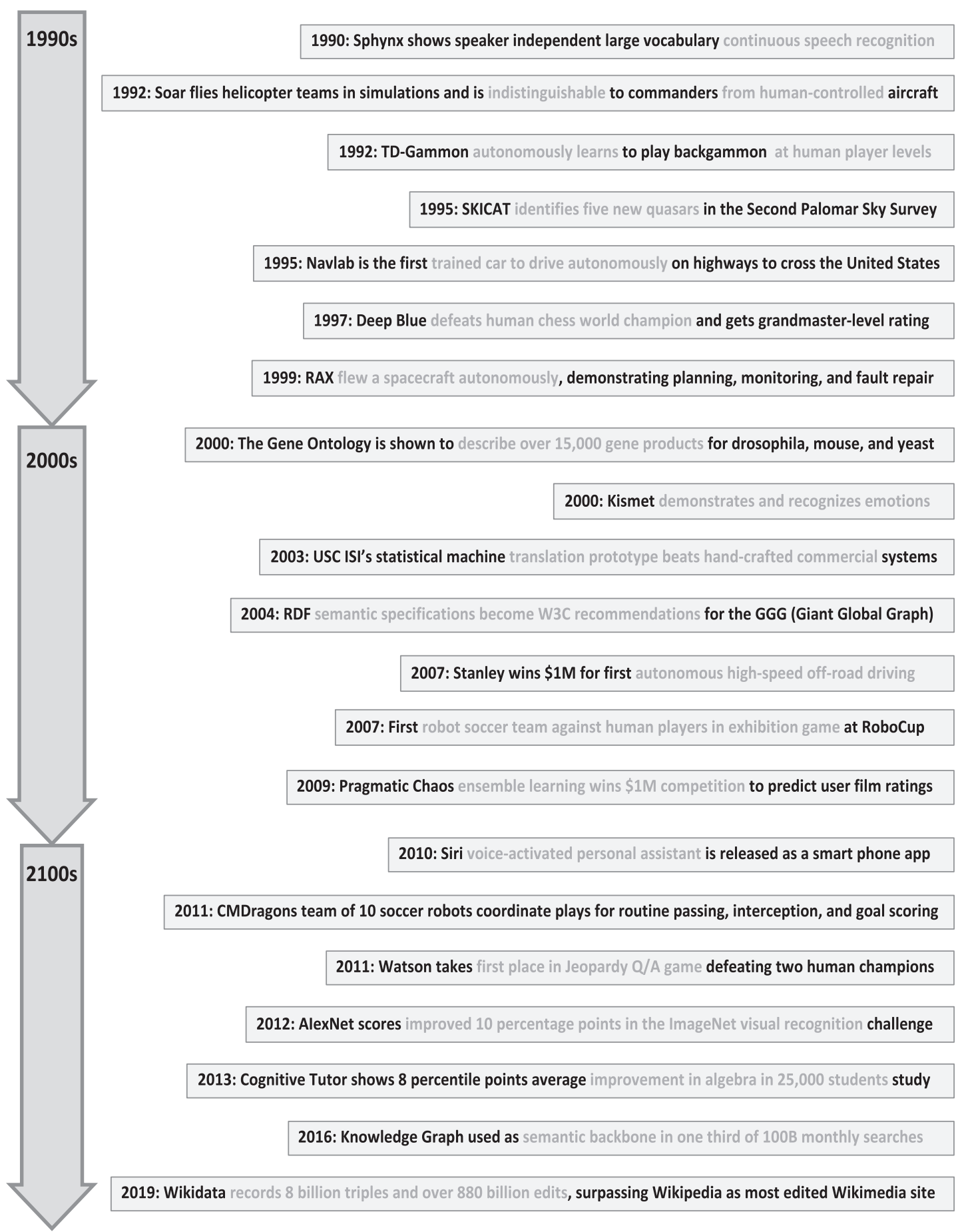

F I G U R E 1 Highlights of significant AI accomplishments over the last few decades, spanning cognitive systems, machine learning, multi-agent systems, knowledge representation, search, planning, robotics, and natural language

shows some of these highlights. In the early 90s, when I was finishing my $\mathrm{Ph}$.D. I used the Sphynx voice recognition system to write my thesis. I thought it was incredible that it worked so well. After graduation, I moved to the Information Sciences Institute at the University of Southern California (USC), where Paul Rosenbloom was collaborating with Allen Newell and John Laird in the SOAR architecture that I mentioned earlier, using it to fly helicopters in teams that a commander found to be indistinguishable from human pilots. I thought that was remarkable. Also during those years, the TD-Gammon system was learning to play backgammon with itself and performing at human levels. SKICAT, built at NASA's JPL, was identifying new quasars using data from Sky Surveys. CMU's Navlab was an autonomous vehicle with a neural network at its core that learned enough to drive itself across the US. Deep Blue was 
able to beat the World Chess Champion, a feat that Kasparov himself has said was eventually inevitable. In 1999, RAX flew in a spaceship and generated plans for the use of its instruments and becoming the first intelligent system to reach outer space. It was an amazing decade for AI.

In the 2000s, many more significant accomplishments would come from all areas of AI. I was excited to see ontologies become very popular, with the Gene Ontology starting to be used to describe thousands of gene products and becoming the core connector for most biology knowledge sources. Kismet demonstrated emotions embodied in an interactive robot. In 2003, a statistical machine translation system beat the performance of manually coded commercial-grade systems that had been built over many years. RDF, a knowledge representation language that become a standard for the Giant Global Graph (the next generation of the World Wide Web), was the initial seed for today's widely used knowledge graphs. Stanley won a million-dollar challenge for off-road autonomous driving. The first robot soccer exhibition game against humans took place, demonstrating teamwork in a highly dynamic environment. At the end of that decade, an ensemble learning system won a million dollars for predicting user movie ratings.

The past decade has also seen many exciting AI accomplishments, and I will just mention them briefly since they are more present in our minds. In 2010, Siri was released as a smart phone app and soon became an integral part in the lives of many mobile phone users. Robot soccer teams were now passing and intercepting across 10 robots coordinating together. Watson won the popular Jeopardy questionanswering contest against the best human players. Alexnet raised the bar tremendously on image recognition and contributed to the revival of neural networks that are flourishing today. Cognitive tutors were demonstrated to improve learning for thousands and thousands of students. Knowledge graphs were used to improve a third of the hundred billion searches conducted in a month. Wikidata recorded a billion triples, or logic assertions, and became the largest crowdsourced knowledge base with more edits than its older sister Wikipedia.

They all are incredible accomplishments of our community, and there are many more that I have not covered here. They illustrate that these advances cut across the spectrum of all areas of AI: interaction, collaboration, search, constraint reasoning, sensing, perception, planning, robotics, knowledge systems, learning, and so on. All across the board we have been able to make tremendous progress.

This message of diversity and breadth is very important for the AI community: that we have accomplished things that the rest of the world may or may not have noticed but that we know place us on solid footing to tackle future problems. All this progress gives us hope that $\mathrm{AI}$ is at the core of new approaches to tackle humanity's crucial challenges, from science to health to innovation to education to policy.

\section{THE IMPERATIVE FOR AI IN SCIENCE}

Accelerating scientific advances is an important grand challenge of our time. In the case of science in particular, I believe that it is not just useful to have AI. AI has indeed become an imperative for science.

There is a long history of AI in science. As early as the 1950s, Herb Simon looked at cognitive aspects of how scientific discovery occurs, and over the years he had many collaborators demonstrating and replicating scientific discoveries. Ed Feigenbaum, who was his student, went off to Stanford to collaborate with Joshua Lederberg and Bruce Buchanan on molecular biology and molecular discovery. There is a long tradition in AI in science. In many cases, the work has been naturally focused on machine learning. And there is great work on analyzing how scientists approach new discoveries in terms of mechanism design, causality, and paradigm shifts.

At the same time, it is important to recognize the human limitations that curb scientific progress (Gil 2017). When we write papers and when scientists look at the world, sometimes there are errors and biases, there is poor reporting in papers, and sometimes the work is not systematic or complete. I will mention, for example, work by Liz Bradley and colleagues on analyzing paleoclimate data where their AI system generated a range of hypotheses and some had not appeared in published papers. Scientists considered them to be valid hypotheses, but they chose not to mention them. As we can see, sometimes, important hypotheses are left not discussed or explored. There are many cases of errors because scientists are human and make mistakes, and so we read about published papers being retracted after some scrutiny about the results. I use the example of a graduate student trying to reproduce the results of a paper by two influential economists, and finding out that they had made an error by omitting some of the data in their tables. Another important problem is that reproducing the results of a published paper is often very challenging, because authors do not include enough information that is crucial to understand how their methods actually worked. These are all important issues that illustrate how human limitations can curtail scientific progress and that I believe $\mathrm{AI}$ is in a great position to address.

At the same time, there is very exciting work that is more recent that I wanted to share with you on AI in science. The computational sustainability community is developing new approaches in multi-agent systems, constraint reasoning, machine learning, and optimization to address a 
range of environmental problems (e.g., Gomes et al. 2019). There is also very important work on materials discovery, where from automated text extraction from published articles they are able to identify particular molecules, recreate the periodic table, and actually predict discoveries that have occurred in the past just from looking at the trends in the literature (e.g., Tshitoyan et al. 2019). There is also increasing value for knowledge-based biomedical data science on how knowledge is used to make progress in life sciences (e.g., Callahan et al. 2020). There are discoveries through machine learning, one of note is on protein folding prediction which incorporated physical and geometrical constraints to outperform any other algorithms and even years of work invested by some labs dedicated to specific proteins (e.g., Senior et al. 2020). It is very exciting to see AI research leading to these advances. I want to point out that these advances come from a diversity of areas in AI. Given the formidable science challenges that our generation faces - understanding the brain, preserving our planet, deciphering the origins of the universe - I believe we need the diversity and breadth of research areas in AI to make strides on these frontiers.

\section{CAPTURING SCIENTIFIC KNOWLEDGE}

In the last two decades, I have worked with scientist in many domains as well as AI researchers in diverse areas on capturing scientific knowledge and using that knowledge to advance science. I will describe first our work on capturing scientific knowledge, then I will discuss how to use it for systematic analyses and for interdisciplinary science.

When you think of scientific knowledge, you think of physics, mathematics, biological processes, and so on. In our research, we take a step back and focus instead on the compositionality and modularity of scientific artifacts that already capture that knowledge. We take models in science as something that we can use as building blocks. For example, a hydrology model can use sophisticated physics equations to represent the movement of rain water in a complex ecosystem. We think that AI systems do not necessarily need to understand exactly how a model works, but instead it needs knowledge about how to use that model to make estimates and predictions. We think of models and other scientific artifacts as modular computational objects - that is a very important concept for us. We focus on the knowledge needed to use them as modular objects: we want to represent the input data they require, the physical variables that they model, the constraints for their use, when their assumptions are appropriate for a particular problem, the parameters that we would like to adjust, and the inter- ventions or changes that we want to do on a situation in order to improve outcomes.

We have worked on a range of projects over the years that touch on different aspects of capturing or representing knowledge. I will discuss here a few of them.

\section{Controlled crowdsourcing of science ontologies}

The first one I will describe is on crowdsourcing vocabularies to describe scientific data and create metadata annotation standards (Gil et al. 2017b). We collaborated with Julien Emile-Geay and Deborah Khider of the USC, and with Nick McKay of Northern Arizona University. This is very interesting work, because in lot of disciplines it takes a lot of effort to agree to standard vocabularies that can be used to describe scientific data. This is the case with paleoclimate, where scientists study past climates in the last few hundred or thousand years by studying very diverse data. They drill cores in different locations on Earth, just to see what happened many years ago through what is buried in the ground. Some scientists drill in the ice, and study the size of the trapped air bubbles. Others look at marine cores and study the remains of coral that appear and how it grew based on the climate at that time. Others drill on lakes to study sediments. It is very challenging to develop a standard way to represent all this data. As a result, it takes them years to analyze all this diverse data in a consistent way to create a model of past climate at planetary scale.

We developed a new approach that we call controlled crowdsourcing. Scientists describe the datasets that they are using as they carry out their work, and they are each asked to propose terms that they would like to use for their own data. They can choose to adopt the terms proposed by others, and pretty soon you have convergence at least for some of the terms. We placed an editorial process on top, very much following the footsteps of Wikipedia, but focused on deciding on terms that are worthy of more general adoption by the community and eventually turning them into ontologies. Users will describe their data as they go, continuously adding what we call crowd properties to extend a very solid set of core ontologies that we either reused or created.

We had to address the challenges of living with an evolving ontology. The system started with an ontology (i.e., the current standard), scientists would annotate their data and propose new properties to describe it, and eventually we had a new extended ontology (i.e., a crowdsourced ontology). However, we could not just replace the original ontology with the new extended ontology, because we had many datasets already annotated using the original one that had to be updated to the new one. We were able to address 
this with AI techniques from ontology development and for non-monotonic reasoning.

The resulting standard for describing paleoclimate data emerged over a period of 2 years, and there was great convergence on how to describe the different datasets as well as a few basic terms that were adopted by all (Khider et al. 2019). I should mention it was accomplished with zero face to face meetings, just a single meeting was held at the very beginning to agree to the overall process. Thanks to AI we were able to enable this standard that has been adopted by the community to develop community infrastructure for efficient data integration and analysis.

It is important to realize that AI was used to synthesize new scientific knowledge which did not exist before, namely vocabulary to describe data across the broad paleoclimate discipline.

\section{Representing scientific workflows}

Another project that I would like to discuss is capturing knowledge about data analysis processes as semantic workflows in our WINGS workflow framework (Gil et al. 2011). Workflows represent multi-step computational methods in science that are repeated and are re-used often. We view them as plans and study them with AI techniques to reason about goals and effects, execution monitoring and replanning, failure detection and recovery, and abstractions. This provides a very powerful framework for many science processes and data analysis methods described in the methods sections of scientific papers.

We do not just treat workflows as computations, instead we treat them as objects of science that have meaning and purpose and we attach to them semantic annotations and constraints. Every constituent of the workflow, whether data or computational step, has an identifier and we can make assertions and express constraints about them. We can assert that a certain type of input data has a property, and that having that property makes it compatible with some analysis step performed downstream in the workflow. We can attach to a workflow a lot of such constraints. These constraints allow us to reason about how to set parameters of specific method steps so they are customized to the data, to reason about generating metadata for the workflow outputs, to reason about how to choose an implementation among many available for a given workflow step, and how to validate that the overall workflow is appropriate for the data at hand.

Figure 2 shows an example workflow constraint. The left side shows the metadata that we have for any data set, and the right shows the step using that type of data as well as other data. A constraint indicates that if two different steps are used together, in this case alignment and assembly, they have to use the same reference genome.

Semantic workflows also use abstraction, so a given step could be executed with different algorithms and implementations. We can very easily reason about abstract methods in science versus specific implementations. Through AI, we are able to carry out very powerful reasoning for composition and exploration of these workflows. We can take a very simple high-level workflow and elaborate it to add many sub-steps. It is basically what some of us in AI would recognize as skeletal planning. Starting with a skeletal plan with high-level steps, we can specialize each step to the current input data based on the constraints that we have about each option for implementing the step.

We capture many scientific methods as semantic workflows. For example, we created a library of workflows for population genomics, and we were able to reproduce papers for which we were able to access the original data. We obtained the same significant results that the papers did, just by reusing workflows from that library. Our workflows used open source software, while some of the papers used proprietary software. Our workflows could use more modern algorithms, while some of these papers used very old algorithms that were known at the time. We were able to obtain the same results because we are using very powerful AI techniques to capture sophisticated scientific methods.

Semantic workflows also enable us to use machine learning to detect common workflow fragments that scientists use with different data, and we demonstrated this with a large collection of neuroimaging workflows (Garijo et al. 2014). This is work with Daniel Garijo here at USC and Oscar Corcho of the Polytechnic University of Madrid. We were able to access hundreds of workflows created manually by scientists and found common workflow fragments that scientists use about how the warping of brain images is done, and general ways in which they approach neuroimaging analysis. To accomplish this, we extended process-mining techniques to exploit the semantic annotations in the workflows, essentially treating them like labeled graphs where we could then map steps across them and create generalizations of any specific workflow fragment. We are using AI to synthesize a new form of scientific knowledge as commonly used abstract workflow fragments that had not been detected before and can now be reused for future neuroimaging analyses.

\section{Provenance and open science}

The last project that I will mention in terms of capturing scientific knowledge is recording provenance. Provenance 


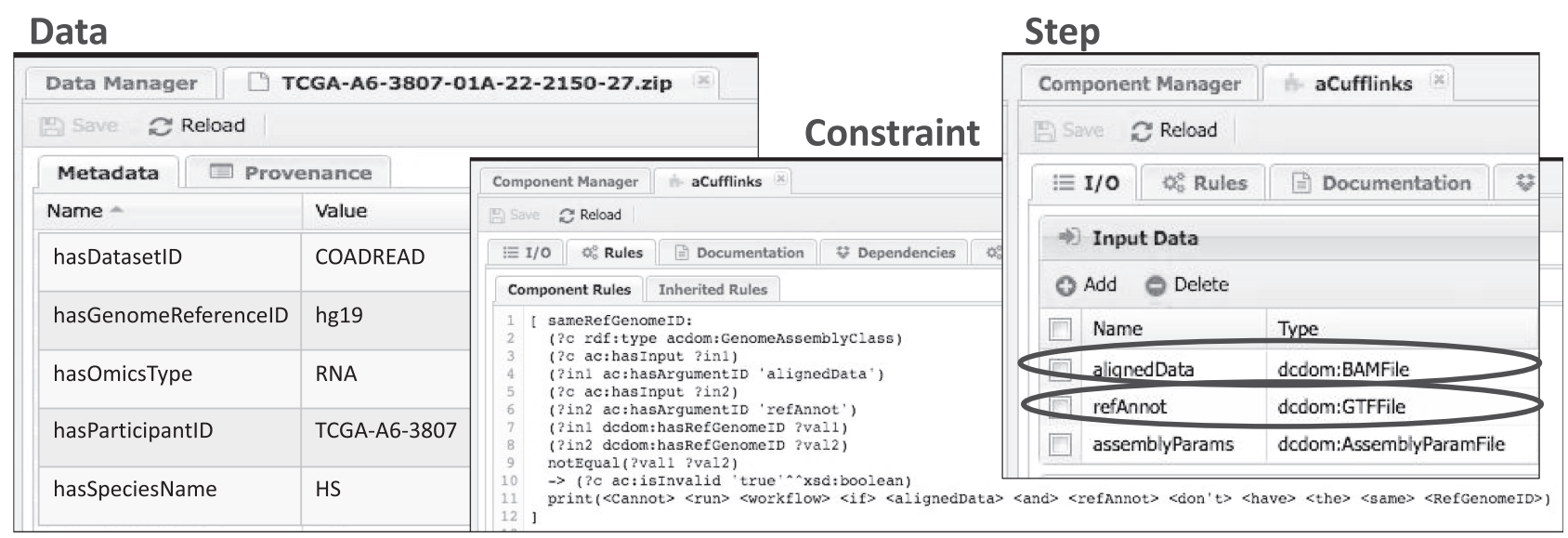

F I G U R E 2 Semantic workflows in WINGS capture constraints about science methods and datasets, enabling the automatic elaboration of high-level methods to customize them to the characteristics of the given data

represents how scientific workflows, as any plans do, once executed leave a trail of the steps that were carried out and the results that they generate. If you have a new dataset that results from an analysis, its provenance record has a very similar underlying structure to the provenance of a dataset collected through a sensor. You can also see that all of the steps that were followed to collect or to prepare data has a lot of similarities to the way that humans actually put together any other digital resource. It is also similar to the way that we describe how a piece of art is generated by a painter or an artist, then years later may go to a curator that cleans it up, and then eventually appear for sale at a gallery. There is a provenance trail for datasets just like there is for pieces of art. A provenance record refers to agents, plans, objects, actions, successful and failed executions, and many other abstractions that we have been studying in AI for decades and designing sophisticated representations and abstractions for them. We worked closely with dozens of people representing diverse disciplines to create a general representation and ontology for provenance, which became a World Wide Web Consortium (W3C) Recommendation, and we are very proud that it has been widely adopted (Moreau et al. 2013).

How would we describe best the science findings and their provenance? We started examining this question with a group of visionary early career researchers to define the geoscience paper of the future (Gil et al. 2016), other groups followed with desiderata for the geophysics paper of the future (Broggini et al. 2017) and the neuroscience paper of the future (Poldrack et al. 2017). We have now generalized the desiderata for scientific papers of the future. I would like to convince all of us scientists to write better papers, because if we do that then the AI systems for machine reading and text extraction will work better. If we do that, AI systems (and other scientists) can actually be in a better position to understand our papers and reproduce the results.

Recently, with Odd Erik Gundersen of Norwegian University of Science and Technology and David Aha of the Naval Research Laboratory, we looked at what the AI paper of the future would look like and proposed core principles for reproducibility of AI publications (Gundersen, Gil, and Aha 2018). I believe it is very important that as a field we formalize more how to capture knowledge properly in our publications. This is not just for the benefit of other researchers. This will benefit AI systems, because the papers we are writing today are too hard for automated extraction and reproducibility. AI systems will be able to read and understand scientific publications once we write them properly providing all the information postulated in these principles. I believe that this will facilitate the development of AI systems for science that reproduce and extend scientific findings, and eventually write papers about their own findings.

That is a quick overview of our work on capturing scientific knowledge, and I want to remark that a lot of the scientific knowledge that we have captured did not exist beforehand. I described our use of AI to synthesize new forms of scientific knowledge as fundamental metadata properties to describe paleoclimate data. I described how we represent scientific methods as semantic workflows, using AI techniques to synthesize new forms of scientific knowledge as commonly used workflow fragments. I mentioned how we use general concepts from AI to create generalized representations of provenance for very diverse types of scientific findings, so our publications really capture the provenance of new results.

Once we capture all this knowledge, how do we use it for science? I will describe two key aspects of how we use this knowledge: systematic analyses and interdisciplinary research. 


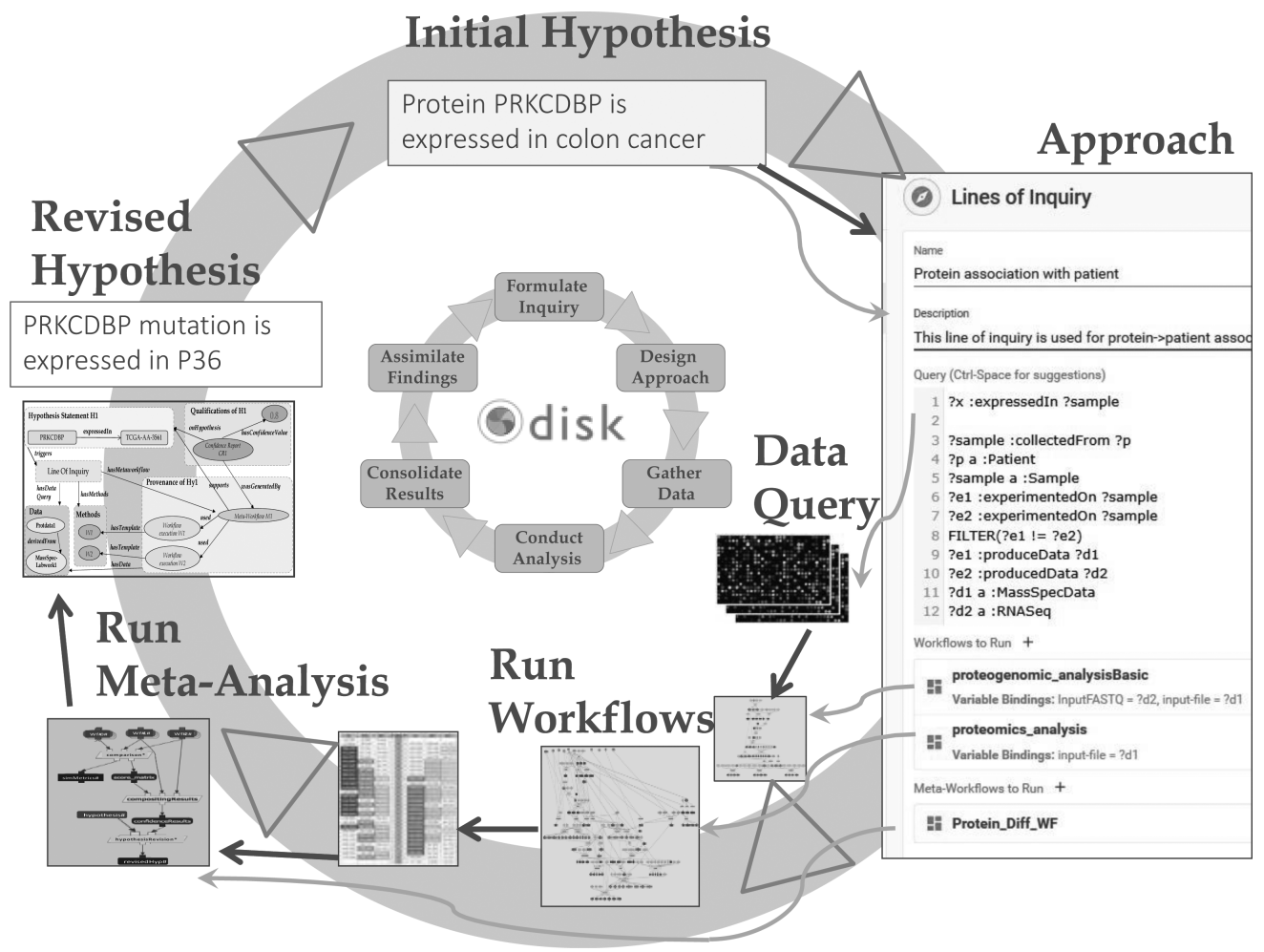

F I G U R E 3 Overview of DISK, an autonomous system for hypothesis-driven discovery that relies on lines of inquiry specified by scientists about the datasets and methods they seek when they pursue specific kinds of hypotheses or questions

\section{AI FOR SYSTEMATIC SCIENTIFIC DATA ANALYSIS}

Our first major use of the scientific knowledge we capture is to carry out systematic scientific data analysis.

We are developing a framework, called DISK, to make hypothesis testing and data analysis more systematic (Gil et al. 2017a). We look at the discovery cycle, starting with formulating new hypotheses, determining what type of data and method can be used to test it (we call this a line of inquiry), retrieving the data from a shared repository, analyzing the data, and then revising the hypothesis. We have automated this hypothesis-driven discovery cycle in our DISK project, a collaboration with Parag Mallick and his group at Stanford University. We work in particular in cancer omics, and there is extensive data in shared repositories that is continuously growing, and therefore we do not need to collect data ourselves.

Figure 3 illustrates how our approach works. A scientist has a hypothesis such as whether a protein is expressed in a certain type of cancer. The line of inquiry indicates that both proteomic and genomic data would be useful for this kind of hypothesis, so DISK executes a query to The Cancer Genome Atlas (TCGA) and to the Clinical Proteomics Tumor Analysis Consortium (CPTAC) data repositories to retrieve data from patients who have that type of cancer. The line of inquiry then indicates that a proteomics work- flow should be run with the proteomics data, a genomics workflow with the genomics data, and a proteogenomic workflow with both types of data together. Then the line of inquiry suggests a meta-workflow to combine the results. After executing the query, the workflows, and the metaworkflow, DISK notifies the scientist that it found evidence that the gene was expressed - but suggested a revised hypothesis that the gene that was expressed was a mutant form of the original hypothesized protein.

These workflows integrate knowledge across disciplines. For example, we use workflows that combine transcriptomics, genomics, and proteomics. Typically, this knowledge is spread across different labs and often different institutions. In addition, there is some analysis needed to ensure that the combination of all of these methods is coherent. This type of knowledge to combine and validate analytic processes is very difficult to obtain and it takes a lot of effort. Once we have captured all of this knowledge, it can be reused very broadly just by re-running these workflows. A great challenge for science today is the compartmentalization of all that knowledge: scientists that collect patient data from genomics and proteomics instruments do not necessarily have expertise in both. A lot of data just sits there waiting to be studied for lack of in-house expertise or collaborators. This presents a tremendous opportunity for AI systems to analyze tirelessly and thoroughly to extract as many findings as possible. 


\section{Representing hypotheses}

DISK represents a hypothesis statement as a triple that expresses a relationship between two objects, for example, that a protein (first object) is associated with (the relationship) a cancer type (second object). Each hypothesis statement has a qualifier that indicates the confidence on the hypothesis, and an evidence trail of the analysis details (that is, the executed workflow) that led to that confidence level. When more data becomes available, it will run a new analysis and revise both the confidence level and the hypothesis. In this case, it will re-examine not just the association of the protein with colon cancer, but it will look for a more specialized and refined hypothesis regarding a subtype of colon cancer. DISK has a hypothesis ontology to express hypothesis statements, qualifiers of confidence, analytical evidence, and the evolution of the statement and its confidence over time.

The way we do science today is that a paper is published with a certain finding and is considered final. However, as more data becomes available, one may wonder if that finding may be different than what was originally discovered. Perhaps the confidence is greater or the evidence more diverse or the hypothesis more precise. We envision AI systems like DISK that will continuously update findings. This would revolutionize our current approach to scientific publications, which are currently static and are considered final once they see the light.

We used DISK to reproduce a seminal cancer omics paper by Bing Zhang and colleagues as part of the CPTAC collaboration (Zhang et al. 2014). The paper's major result was the discovery of a new, fifth subtype of colon cancer based on patient proteomes. It took 2 years to carry out this large-scale proteogenomic study. All the data is available, and the paper has many figures that show intermediate results and a long appendix with many details. Despite that, it took us quite a long time to understand what was done and reproduce the results. Using DISK, we were able to reproduce the results and find the same new fifth subtype of colon cancer.

\section{Reproducing and extending science findings}

We found that even though DISK was able to independently discover a fifth subtype and confirm the main finding, it obtained very different intermediate results. DISK obtained very different results for the peptides and proteins that were present in each patient's tumors. It found a 10 percent difference. This is quite large. After discussion with the authors, we discovered they had used mul- tiple thresholds and complex filtering in their analysis. In particular, they used a stringent filter at one step, and a less stringent filter in a subsequent step. None of that was mentioned in the paper. Incorporating these filters cut the difference in the results in half, which is still quite a large difference but it is more acceptable. This points to how sensitive intermediate results can be to subtle variations in the methods. The overall finding still stands, but it is quite alarming that the detailed analytic results are so different.

We then used DISK to try a few alternative methods, for example, different approaches to do peptide search. This is very easy to do using semantic workflows, since DISK can easily explore all the methods that fit a step and find the best performing ones. We found that there was a 35 percent difference in the protein identifications, and that key proteins were missed by some of the methods. This is alarming, because proteogenomics publications typically use only one search method. Many proteins are likely to be missed unless we use asn AI system like DISK to perform a more systematic exploration of alternative methods.

DISK also showed a marked difference when using the same method with different parameters, or different reference databases. The protein and peptide identifications also varied substantially. In addition, we looked at other measures, like single aminoacid variants and variant peptides, and found the differences to be quite substantial. This shows that there is a great need to use AI to systematically explore all these method parameters, and to understand their differences.

I mentioned earlier that another significant opportunity for $\mathrm{AI}$ is to continually analyze data and update findings as new data becomes available. We took the dataset from the original (Zhang et al. 2014) paper, and updated the analysis with the new samples that have appeared in the data repositories since the publication of that original paper. When the first few new samples are added, they fall nicely in the five subtypes. The next few samples do as well, as do the next ones. When we added the last few samples, the clustering algorithm still generated five subtypes but it grouped the samples a bit differently so it is unclear that they are the same five subtypes as before. This makes the analysis more challenging, and one of the things we had to do to revise hypotheses over time is to develop new workflows with batch effect correction for the new samples.

Finally, using AI to carry out systematic analyses makes it easy to apply methods to new datasets. We applied the same workflows for datasets from colorectal cancer (CRC95), NCI60 (nine types of cancer, multiple tissues), ovarian cancer (OV), and breast cancer (BRCA). DISK found, for example, that colorectal cancer has a lot less variance than other types of cancer. This is well known, but we were able to find that very easily very quickly. 
We think DISK can be used as a reference benchmarking framework, and we demonstrated this for the National Cancer Institute CPTAC DREAM Proteogenomics Challenge (Srivastava et al. 2019). For that challenge, the same datasets were analyzed by dozens of teams to identify proteins. It is very hard to pinpoint why a specific team did better, because their methods are quite similar and only differ in very small ways. The teams follow a typical analysis structure, where they first align the gene sequences, then they quantify and normalize the data, and then they predict the protein levels. We created generic semantic workflows that DISK could reason about to specialize them into the kinds of methods adopted by individual teams. With this framework, we can analyze the solutions proposed by different teams, and compare and contrast what specific differences made a method significantly better. We found that gene-specific models give significantly better results, while the other steps are only responsible for minor improvements. In addition, by exploring all the possibilities systematically, DISK found the optimal combination of methods.

All this work illustrates how AI can make a difference in making scientific research more systematic. Every year omics datasets are increasing in size, diversity, and complexity. There is more and more analytic complexity, as new algorithms and methods appear. The expertise is very fragmented. At some point sooner or later, biomedical researchers will not be able to keep up. Imagine if AI systems like DISK were used to analyze proteogenomic data systematically in every cancer study. This would accelerate discoveries for new problems and easily update earlier findings when new data is available. AI will become a game changer for omics research, and many other areas of science where there is abundant data available.

\section{AI FOR INTERDISCIPLINARY SCIENCE FRONTIERS}

The second way in which we use scientific knowledge is in exploring interdisciplinary science frontiers.

We have a project called MINT, for Model INTegration, where we are looking at how climate is affecting water availability, agriculture and food production, socioeconomic factors, with extremes of flooding and drought cutting across all those aspects (Gil et al. 2021). To study these types of problems, we have to integrate climate models, models for hydrology, agriculture models, economics models, and social models. Each of these types of models is developed by different disciplines each with their own methods and approaches. For example, hydrology models use physics equations to simulate the movement of rain water in an ecosystem. In contrast, social models often rely on agent-based simulations of social behaviors. It takes months or years to assemble integrated models for a certain region, since it is truly a craft. There is increasing demand to understand these cross-domain regional phenomena. We have a growing community of collaborators in Ethiopia, Texas, and California.

\section{Integrating models across science domains}

Our goal is to reduce the time to assemble cross-domain models from months or years down to days. To accomplish this, we are using AI to mediate between models at all levels: at the variable level, at the dataset level, at the process level, and so on. At the variable level, we use an ontology conceived by Scott Peckham at the University of Colorado that is very extensive and has thousands of terms. It has not been built manually, instead it was designed using principles for how to describe physical variables in a consistent, uniform way. With this ontology, we can be very precise about what a particular variable in a model is measuring. We think of a model as a computational object, where we need to represent the data formats, the physical variables, the constraints on how to use the model, and so on.

We also use AI to mediate models and datasets from different sources that have varying quality, and this is also a big challenge. We represent what those datasets contain, what variables they characterize, and the structure of the files. Then, we use AI planning techniques to automatically transform the datasets from their native format to the format that a model requires. Craig Knoblock and Jay Pujara at USC have been leading this research. There has been a lot of work in this particular topic over the years, and we are building on that to work with much more complex data.

In some regions, there is simply not enough data available for modeling. This is the case with our work with Dr. Belete Berhanu and colleagues of the Addis Ababa Institute of Technology in Ethiopia. For that region's river basins, there are not enough gauges and measurements of water levels over the last 30 years to calibrate hydrology models. In the case in Ethiopia, if we model the Awash or Baro rivers, the models are incredibly coarse and the visualizations of flooding indicators appear very pixelated. In contrast, in work that we are doing with Suzanne Pierce of the University of Texas at Austin we can show the extent of the flooding building by building in Travis County. And the models actually generate very accurate predictions of the flooding that correspond well to manual flood maps. In order to improve our models for Ethiopia, we use machine learning to extract useful information from satellite data about water levels in rivers, the degree of flooding, what kinds of crops were typically planted over the years and so 


\section{Model Catalog}

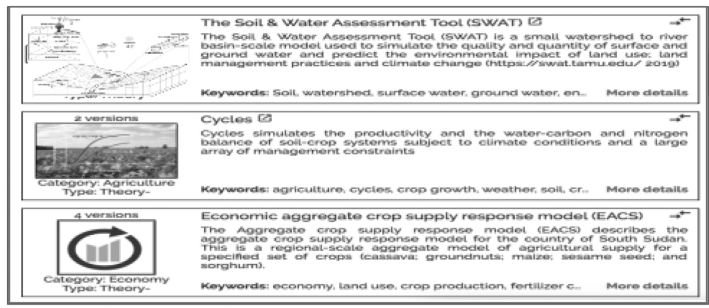

\section{Model Metadata}

\section{Model Metadata} Ontology
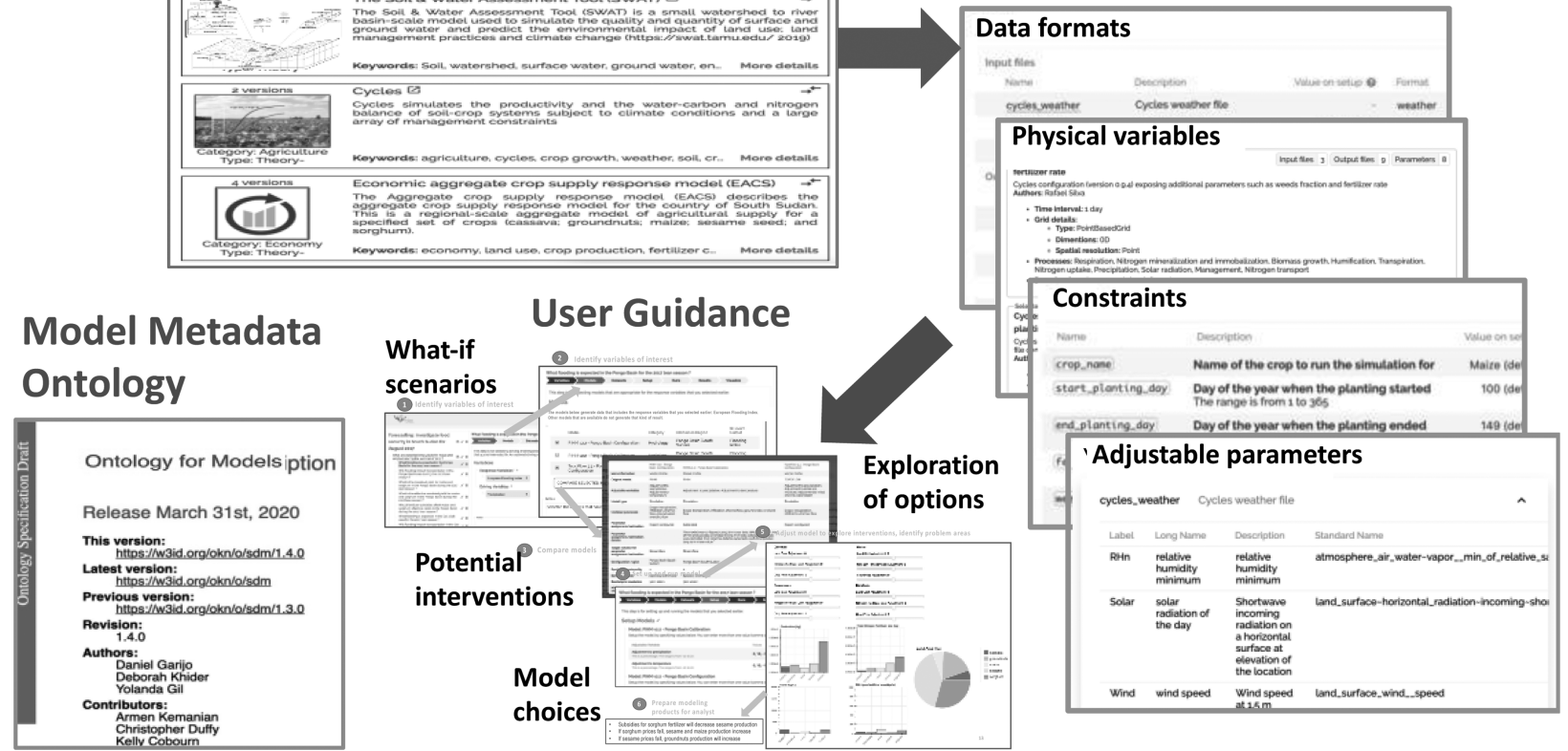

F I G U R E 4 MINT captures metadata and constraints about models and datasets and uses them to guide users to develop and integrate models from different disciplines more efficiently

on. These machine learning techniques incorporate basic physics knowledge as constraints. This is work by Vipin Kumar and his group at the University of Minnesota. There is significant ongoing research in combining physics-based models with machine learning models in a variety of ways, and this is a very promising direction to use AI to improve the accuracy of models in geosciences.

\section{Delivering models to decision makers}

As far as the models themselves, we also use AI to represent abstractions and connect the models to decisions. For this I am going to use examples from an agriculture model, developed by Armen Kemanian at Penn State University. These kinds of models have a lot of detailed parameters, such as nitrogen stress or solar radiation levels. However, somebody making decisions about what crops to plant does not really need to know that. Therefore, we focus on the variables that a decision maker would want to adjust. How much corn should be planted? How much sorghum should be planted? How much irrigation should be used? Those are the kinds of factors that a decision maker would want to change, and then see how different weather predictions affect the outcome of interest, which is the crop yield.

This allows us to use a model in a very compositional way. It is still very challenging, but we have very rich descriptions of what each model can be used for, as illustrated in Figure 4. This is work with Daniel Garijo and
Deborah Khider at USC. Each model has many different variants as we adapt it for different regions, and for each variant we capture the data formats, the physical variables, the use constraints, and the adjustable parameters. We have developed ontologies for describing scientific software, and extended them to describe models. These descriptions of the models can be used to drive their integration. We can see the areas that will be flooded (from a hydrology model) and how that affects other things: the crops that will be lost if not harvested earlier (from an agriculture model), the roads that will not be trafficable to bring the food where it is needed (from a transportation model), and the towns that people will flee to reach dry grounds.

This work illustrates how AI can integrate diverse knowledge from different disciplines and integrate it to create unified models to understand complex phenomena. AI provides a crucial capability for many problems where we need to characterize complex systems where natural, human, and engineered processes interact, such as environmental and geosciences research.

\section{A PERSPECTIVE ON THE FUTURE}

I have described how we capture scientific knowledge, and illustrated how we use all of this knowledge to perform systematic data analysis and to engage in interdisciplinary science. Now I would like to take a step back, and revisit the question that I posed at the beginning: "Will 
AI write scientific papers in the future?" If we had AI systems with enough knowledge to follow the methods of science, we have to wonder if they could generate new findings on their own and report those findings by writing their own papers. This would liberate scientists from routine work and enable them to spend more time on the creative aspects of science.

Scientific research is becoming more and more complex. Many significant discoveries are the product of collaborations of hundreds or thousands of scientists. The Human Genome Project was an ambitious endeavor that involved hundreds of scientists. The discovery of the Higgs boson in 2012 was reported in an article with more than four thousand authors (Aad et al. 2012). The science questions that we want to ask are more and more complex, and these kinds of discoveries take several years and involve many people. These are very unique kinds of efforts in science today, but I wonder what it would take for AI to help make scientific advances of this caliber more commonplace.

Gary Kasparov made an interesting observation about humans and machines in freestyle chess. Here, a player can be a combination of any number of humans and any number and type of machines. He remarked that they were surprised that a weak human plus a machine plus a better process was superior to a strong computer alone, and superior to a strong human and a machine and a less ideal process. This highlights the importance of organizing and distributing work appropriately that defining a good process is crucial when combining the skills of humans and machines together.

What would it take for machines to collaborate with scientists in similarly powerful teams? In the case of chess, an AI system does not need any special skills to become a team member since the only requirement is to communicate to others the recommended next move. In contrast, for an AI system to be a collaborator in science I believe that it needs to have very sophisticated skills. I recently proposed a set of principles for the design of thoughtful AI systems that will be good partners for a scientist (Gil 2017). Today, a researcher gives an AI system some data, an algorithm, and the function to optimize. There is a lot of research that needs to be accomplished so that an AI system can be a partner. The principles that I have proposed for thoughtful AI systems, as summarized in Table 1, include:

- Rationality principle: Their behavior to accomplish tasks should be governed by the knowledge in the AI system. That is, there is deliberation and understanding through knowledge about the task.

- Context principle: Seek knowledge and resources that would be considered important about the context of a given task, particularly to handle difficult or unusual
TA B L E 1 Principles for thoughtful AI systems that will partner with scientists to accelerate discoveries (from [Gil, 2017])

\begin{tabular}{l|l}
\hline Principles for thoughtful AI systems \\
\hline $\begin{array}{l}\text { Rationality principle } \\
\text { Context principle }\end{array}$ & $\begin{array}{c}\text { Behavior is governed by knowledge } \\
\text { significance of tasks }\end{array}$ \\
\hline Initiative principle & $\begin{array}{c}\text { Proactively new learn knowledge } \\
\text { relevant to a task }\end{array}$ \\
\hline Network principle & $\begin{array}{c}\text { Access external sources of } \\
\text { knowledge and capabilities }\end{array}$ \\
\hline Articulation principle & $\begin{array}{c}\text { Respond with persuasive } \\
\text { justifications and arguments }\end{array}$ \\
\hline Systems principle & $\begin{array}{c}\text { Facilitate integration and } \\
\text { compositionality with other } \\
\text { systems }\end{array}$ \\
\hline Ethics principle & $\begin{array}{c}\text { Behavior that conveys limitations, } \\
\text { uncertainty, and unknowns }\end{array}$ \\
\hline
\end{tabular}

cases. That is, their knowledge is not confined to the scope of accomplishing a specific task.

- Initiative principle: Learn new knowledge proactively, and can use a variety of mechanisms to acquire it (e.g., being taught by others, learn from data, extracted from text, obtain by experimenting with the world, etc.). That is, they are not just passive recipients of data or knowledge that is selected and prepared for them by people.

- Network principle: Connect to a variety of resources online (documents, services, sensors, and effectors) as well as people. This enables the ability to independently access new knowledge and new capabilities.

- Articulation principle: Understand guidance and questions posed to them and respond not just with appropriate behavior and answers but also with responses that may include refining or changing the question, describing different approaches to answer it, describing and justifying an answer, and comparing alternative answers or approaches.

- Systems principle: Exhibit basic engineering design properties (such as compositionality, abstraction, and connectivity) that support integration with other systems.

- Ethics principle: Incorporate responsible and ethical behaviors, in particular the ability to recognize and convey uncertainty and limitations in making decisions and taking action.

Thoughtful AI systems that adopt these principles would provide a solid basis for developing AI scientists.

Will AI write scientific papers in the future? I am certainly working on many projects in that direction, and many others are working in relevant areas. There is a lot of good work on re-imagining the future of science and 
research. We have worked with the geosciences community to envision a future with AI (Gil et al. 2019). I will highlight Hiroaki Kitano's recent article in AI Magazine on AI to win the Nobel Prize (Kitano 2016). I think that is not only quite an ambitious goal but also very exciting. There are a lot of great possible outcomes along the way: AI to reproduce published articles, AI as a research assistant, $\mathrm{AI}$ as a partner, and $\mathrm{AI}$ as co-author.

So, will AI write scientific papers in the future? I will go back to the observation that I made at the very beginning of this talk: the AI community has always been visionary, broad, inclusive, interdisciplinary, determined, and successful at challenging endeavors so the answer would be affirmative. At the same time, I showed that human scientists are not systematic, they make errors, they have biases, and they do poor reporting. This argues that may be AI systems will significantly improve our approach to science and will end up authoring scientific papers with proper documentation and provenance. But at the same time, we need to remember that penicillin came out of a human error, where Alexander Fleming accidentally left the lid open in a culture plate and it ended up contaminated with Penicillium mold that killed the bacteria. So, we need to reflect on the wonders of human ingenuity, and its crucial role in scientific discovery and innovation.

In the end, if we find imaginative ways to combine humans and machines I believe we will see really remarkable outcomes in science. My hope is that this perspective will inspire us all to pursue significant research goals, that if we read an article or work on a problem where we think to ourselves "I could write an AI for that" then we will be less excited to pursue that line of work. But if we read an article and have a sense that only a human could come up with that kind of idea, I am willing to bet that in many cases we will hear things like "Well, I just was stubborn," "I had this firm belief in this possibility," or even "I made a mistake and that led me to see things differently." I do not know what the future will look like as scientists partner with AI to do significant discoveries, but I am very excited to design that future together with all of you.

In this talk, I have focused on science but the kinds of AI research that I discussed are universally applicable to other important areas where AI could be a game changer: wellbeing and quality of life, education, social justice, innovation, and many others. You can find very compelling use cases and interesting research directions in a recent community roadmap for the next 20 years of AI research (Gil and Selman 2019). Please continue to push for the breadth and diversity of AI. All of your ideas, every single one, are important for a future where AI changes the way that humanity approaches the most formidable challenges.

\section{ACKNOWLEDGEMENTS}

I would like to thank my collaborators over the years, especially (and in no particular order) Varun Ratnakar, Daniel Garijo, Deborah Khider, Maximiliano Osorio, Hernan Vargas, Jihie Kim, Paul Groth, Ewa Deelman, Karan Vahi; Rafael Ferreira, Rajiv Mayani, Craig Knoblock, Jay Pujara, Kevin Knight, Chris Mattmann, Paul Ramirez, Dan Crichton, Ralph Bergmann, William Cheung, Oscar Corcho, Pedro Gonzalez, Ricky Sethi, Carole Goble, Natalia Villanueva, Deanna Pennington, Julien EmileGeay, Nick McKay, Felix Michel, Matheus Hauder, Paul Hanson, Jordan Read, Scott Peckham, Maria Stoica, Chris Duffy, Armen Kemanian, Vipin Kumar, Neda Jahanshad, Phil Bourne, Chris Mason, Joel Saltz, Tahsin Kurk, Jill Mesirov, Michael Reich, Shannon McWeeney, Christina Zhang, Parag Mallick, Ravali Adusumilli, Hunter Boyce, Tom Harmon, Sandra Villamizar, Suzanne Pierce, Cédric David, Ibrahim Demir, Jonathan Goodall, Bakinam Essawy, Robinson Fulweiler, Allen Pope, Mimi Tzeng, Odd Erik Gundersen, and so many others. My thanks also go to Kevin Knight for coming all the way to hear me talk for the millionth time, and to Victoria Knight for patiently transcribing the original recording. I am very grateful for the sponsorship of my work over the years from the National Science Foundation, the Defense Advanced Research Projects Agency, the National Institutes of Health, the Intelligence Advanced Research Projects Activity, the National Aeronautics and Space Administration, the Air Force Office of Scientific Research, the Office of Naval Research, Elsevier, the Bill and Melinda Gates Foundation, J.P. Morgan Chase, and the Kavli Foundation.

\section{REFER EN C ES}

Aad, G., et al. 2012. "Observation of a new particle in the search for the Standard Model Higgs boson with the ATLAS detector at the LHC.” Physics Letters B 716(1): 1-29. https://doi.org/10.1016/j. physletb.2012.08.020

Broggini, F., J. Dellinger, S. Fomel, and Y. Liu. 2017. "Reproducible research: Geophysics papers of the future - Introduction.” Geophysics 82(6): WBi-WBii. https://doi.org/10.1190/ geo2017-0918-spseintro.1

Callahan, T. J., I. J. Tripodi, H. Pielke-Lombardo, and L. E. Hunter. 2020. "Knowledge-based biomedical data science." Annual Review of Biomedical Data Science 3(1): 23-41. https://doi.org/10.1146/ annurev-biodatasci-010820-091627.

Garijo, D., O. Corcho, Y. Gil, B. A. Gutman, I. D. Dinov, P. M. Thompson, and A. W. Toga. 2014. "FragFlow: Automated fragment detection in scientific workflows." In Proceedings of the IEEE Conference on e-Science, Guarujua, Brazil. https://doi.org/10.1109/ eScience.2014.32

Gil, Y., P. A. Gonzalez-Calero, J. Kim, J. Moody, and V. Ratnakar. 2011. "A semantic framework for automatic generation of computational workflows using distributed data and component catalogs." Journal of Experimental and Theoretical Artificial Intelligence 23(4): 389-467. https://doi.org/10.1080/0952813X.2010.490962 
Gil, Y., C. H. David, I. Demir, B. T. Essawy, R. W. Fulweiler, J. L. Goodall, L. Karlstrom, et al. 2016. "Towards the geoscience paper of the future: Best practices for documenting and sharing research from data to software to provenance." Earth and Space Science 3(10): 388-415. http://doi.org/10.1002/2015EA000136

Gil, Y. 2017.“Thoughtful artificial intelligence: Forging a new partnership for data science and scientific discovery." Data Science 1(1): 119-29. http://doi.org/10.3233/DS-170011.

Gil, Y., D. Garijo, V. Ratnakar, R. Mayani, R. Adusumilli, H. Boyce, A. Srivastava, et al. 2017a. "Towards continuous scientific data analysis and hypothesis evolution." In Proceedings of the Thirty-First AAAI Conference on Artificial Intelligence (AAAI-17), San Francisco, CA. Retrieved from https://ojs.aaai.org/index.php/AAAI/ article/view/11157

Gil, Y., D. Garijo, V. Ratnakar, D. Khider, J. Emile-Geay, and N. McKay. 2017b. "A controlled crowdsourcing approach for practical ontology extensions and metadata annotations.” In Proceedings of the Sixteenth International Semantic Web Conference (ISWC), Vienna, Austria. http://doi.org/10.1007/978-3-319-68204-4_24

Gil, Y., and B. Selman, eds. 2019. “A 20-year community roadmap for artificial intelligence research in the US.” In Computing Community Consortium (CCC) and Association for the Advancement of Artificial Intelligence (AAAI) report. Published in arXiv, https: //arxiv.org/abs/1908.02624v1.

Gil, Y., S. A. Pierce, H. Babaie, A. Banerjee, K. Borne, G. Bust, M. Cheatham, et al. 2019. "Intelligent systems for geosciences: An essential research agenda." Communications of the ACM 62(1): 7684. http://doi.org/10.1145/3192335.

Gil, Y., D. Garijo, D. Khider, C. A. Knoblock, V. Ratnakar, M. Osorio, H. Vargas, et al. 2021. "Artificial intelligence for modeling complex systems: Taming expert models to improve decision making." In ACM Transactions on Interactive Intelligent Systems (TiiS). 11(2) pp. 1-49. https://doi.org/10.1145/3453172

Gomes, C., T. Dietterich, C. Barrett, J. Conrad, B. Dilkina, S. Ermon, F. Fang, et al. 2019. "Computational sustainability: Computing for a better world and a sustainable future." Communications of the ACM 62(9): 56-65. https://doi.org/10.1145/3339399.

Gundersen, O. E., Y. Gil, and D. W. Aha. 2018. “On reproducible AI: Towards reproducible research, open science, and digital scholarship in AI publications." AI Magazine 39(3): 56-68. http://doi.org/ 10.1609/aimag.v39i3.2816

Khider, D., J. Emile-Geay, N. P. McKay, Y. Gil, D. Garijo, V. Ratnakar, M. Alonso-Garcia, et al. 2019. "PaCTS 1.0: A crowdsourced reporting standard for paleoclimate data." Paleoceanography and Paleoclimatology 34(10): 1570-96. http://doi.org/10.1029/2019PA003632

Kitano, H. 2016. "Artificial intelligence to win the nobel prize and beyond: Creating the engine for scientific discovery." AI Magazine 37(1): 39-49. https://doi.org/10.1609/aimag.v37i1.2642.

Moreau, L., P. Missier, K. Belhajjame, R. B'Far, J. Cheney, S. Coppens, S. Cresswell, et al. 2013. "PROV-DM: The PROV data model." In W3C Recommendation. Available from http://www.w3.org/TR/ prov-dm/

Poldrack, R. A., C. I. Baker, J. Durnez, K. J. Gorgolewski, P. M. Matthews, M. R. Munafò, T. E. Nichols, et al. 2017. "Scanning the horizon: Towards transparent and reproducible neuroimaging research." Nature Reviews Neuroscience 18(2): 115-26. https: //doi.org/10.1038/nrn.2016.167.

Senior, A. W., R. Evans, J. Jumper, J. Kirkpatrick, L. Sifre, T. Green, C. Qin, et al. 2020. "Improved protein structure prediction using potentials from deep learning." Nature 577: 706-10. https://doi. org/10.1038/s41586-019-1923-7.

Srivastava, A., R. Adusumilli, H. Boyce, D. Garijo, V. Ratnakar, R. Mayani, T. Yu, et al. 2019. "Semantic workflows for benchmark challenges: Enhancing comparability, reusability and reproducibility." Proceedings of the Pacific Symposium on Biocomputing (PSB), Waimea, HI. https://doi.org/10.1142/9789813279827_ 0019

Tshitoyan, V., J. Dagdelen, L. Weston, A. Dunn, Z. Rong, O. Kononova, K. A. Persson, et al. 2019. "Unsupervised word embeddings capture latent knowledge from materials science literature." Nature 571: 95-8. https://doi.org/10.1038/s41586-019-1335-8.

Zhang, B., J. Wang, X. Wang, J. Zhu, Q. Liu, Z. Shi, M. C. Chambers, et al. 2014. "Proteogenomic characterization of human colon and rectal cancer.” Nature 513: 382-7. https://doi.org/10.1038/ nature13438

\section{AUTHOR BIOGRAPHY}

Dr. Yolanda Gil is Director of Data Science and AI Initiatives at the Viterbi School of Engineering, and Senior Director for AI and Data Science Strategy at the Information Sciences Institute of the University of Southern California. She is a Research Professor in Computer Science and in Spatial Sciences. She received her M.S. and Ph. D. degrees in Computer Science from Carnegie Mellon University, with a focus on artificial intelligence. Her research is on intelligent interfaces for knowledge capture and discovery, which she investigates in a variety of projects concerning scientific discovery, knowledge-based planning and problem solving, information analysis and assessment of trust, semantic annotation and metadata, and communitywide development of knowledge bases. Dr. Gil collaborates with scientists in different domains on semantic workflows and metadata capture, social knowledge collection, computer-mediated collaboration, and automated discovery. She is a Fellow of the Association for Computing Machinery (ACM), the Association for the Advancement of Science (AAAS), and the Institute of Electrical and Electronics Engineers (IEEE). She is also Fellow of the Association for the Advancement of Artificial Intelligence (AAAI), and served as its 24th President. More information about her work is available at https://www.isi.edu/ gil/.

How to cite this article: Gil, Y. 2021. "Will AI write scientific papers in the future?" AI Magazine 42: 3-15. https://doi.org/10.1609/aaai.12027 